\title{
Eficácia de herbicidas isolados ou em mistura no controle da planta exótica invasora Urochloa subquadripara
}

\author{
Effectiveness of herbicides isolated or in mixture in control of the exotic \\ plant invasive Urochloa subquadripara
}

Luan Fernando Chiarotti ${ }^{1}$, Pâmela Castro Pereira ${ }^{1}$, Ricardo Henrique da Cruz Poliseli Scannavino ${ }^{1}$, Klara Silva Castro ${ }^{1}$, Wilson Roberto Cerveira Junior ${ }^{2}$, Claudinei da Cruz ${ }^{1 *}$

Resumo - O objetivo deste estudo foi avaliar a eficácia dos herbicidas glyphosate, saflufenacil, imazamox, imazapyr e penoxsulam, isolados e em mistura, no controle de Urochloa subquadripara. Para tanto, em casa de vegetação, cinco ponteiros das plantas foram transplantados em caixas de 2,5 litros contento $5 \mathrm{~cm}$ de solo, areia grossa lavada e composto orgânico (na proporção 1:1:1). Após 30 dias, foi realizada a aplicação dos herbicidas com pulverizador costal à pressão constante de 25 psi mantida por $\mathrm{CO}_{2}$, com consumo de calda equivalentes a $200 \mathrm{~L} \mathrm{ha}^{-1}$. As avaliações foram efetuadas por meio de notas de eficácia aos 3, 7, 15, 21, 30, 45 e 60 dias após a aplicação (DAA). Os herbicidas glyphosate e imazamox, isolados, apresentaram excelente controle (100\%) de U. subquadripara, enquanto que, o imazapyr e o penoxsulam (48 $\left.\mathrm{g} \mathrm{ha}^{-1}\right)$ apresentaram controle satisfatório com 80 a $85 \%$ de eficácia de controle. Os herbicidas glyphosate, imazamox e imazapyr (isolados e em mistura), o saflufenacil + glyphosate (mistura) e o penoxsulam + glyphosate (mistura) podem ser empregados em programas de manejo desta planta aquática invasora.

Palavras-chave: braquiária d’água, controle químico, modelo biológico, plantas aquáticas

Abstract - The objective of this study was to evaluate the efficacy of glyphosate, saflufenacil, imazamox, imazapyr and penoxsulam alone or in combination in the control of Urochloa subquadripara. To do so, in greenhouse, five plant pointers were transplanted into 2.5 liter boxes containing $5.0 \mathrm{~cm}$ of soil, washed coarse sand and organic compound (1:1:1; ratio). After 30 days, the herbicides with costal sprayer were applied at a constant pressure of 25 p.s.i. maintained by $\mathrm{CO}_{2}$, with consumption of syrup equivalent to $200 \mathrm{~L} \mathrm{ha}^{-1}$. The evaluations were performed through efficacy notes at 3, 7, 15, 21, 30, 45 and 60 days after application (DAA). The herbicides glyphosate and imazamox, isolated, showed excellent control (100\%) of $U$. subquadripara, whereas imazapyr and penoxsulam (48.0 $\mathrm{g} \mathrm{ha}^{-1}$ ) presented satisfactory control with 80 to $85 \%$ control efficacy. The herbicides glyphosate, imazamox and imazapyr (isolated and mixed), saflufenacil + glyphosate (mixture) and penoxsulam + glyphosate (mixture) can be used in management programs of this invasive aquatic plant.

Keywords: tannergrass, chemical control, biological model, exotic invasive plant

Recebido: Fevereiro 09, 2018. Aceito: Julho 16, 2018.

${ }^{1}$ Centro Universitário, Fundação Educacional de Barretos, Av. Prof. Roberto Frade Monte, 389, CEP 14783-226, Barretos, SP, Brasil.E-mail: luan_chiarotti@hotmail.com; pereirapamelacastro@hotmail.com; ricardim_polizelli@hotmail.com; claudineicruz@gmail.com

${ }^{2}$ Faculdade de Ciências Agrárias e Veterinárias, Universidade Estadual Paulista - UNESP, Jaboticabal, SP, Brasil. E-mail: wilsonrcjunior@gmail.com 


\section{Introdução}

As invasões biológicas dos ecossistemas aquáticos são um dos principais problemas ambientais no planeta. As espécies invasoras são aquelas que se tornam bem-sucedidas em seu próprio ambiente (nativo), devido a alguma alteração ambiental ou em um novo habitat (exótico) (Li, 2014) e estabelecem pressão sobre as espécies nativas. Os ecossistemas de água doce são vulneráveis aos impactos antropogênicos, apresentando invasões recorrentes e efeitos variados (Strayer, 2010). A maioria das espécies de plantas aquáticas são consideradas invasoras, devido a facilidade de reprodução, dispersão facilitada por propagação vegetativa e adaptação a uma variedade de habitats (Lu et al., 2018).

As plantas aquáticas (incluindo espécies exóticas) tem a capacidade de colonizar a maioria dos habitats aquáticos disponíveis (Riis, 2008). As espécies de Poaceae aquáticas, como a Urochloa subquadripara, têm ocorrido com muita frequência em ecossistemas aquáticos brasileiros (Domingos et al., 2011). A invasão de Urochloa mutica alterou a morfologia e a hidrologia de córregos, com aumento das taxas de sedimentação dos corpos hídricos (Bunn et al., 1998). Outro problema recente é o aumento da taxa de evapotranspiração que ocorre nos corpos hídricos após o estabelecimento das plantas invasoras (Anda et al., 2015).

Além do controle das plantas aquáticas endêmicas, ocorre as invasoras exóticas, como braquiária d'água ou capim-tannergrass (U. subquadripara $=$ Brachiaria arrecta). Esta espécie foi introduzida no Brasil em 1989 como forrageira, porém, rapidamente passou a ocupar várias regiões semialagadas, várzeas e até corpos hídricos de maior volume. Esta planta tornou-se problema nos principais reservatórios de hidrelétricas do país, como Santana, no Rio de Janeiro (Pitelli et al., 2008) e Barra Bonita, São Paulo (Domingos et al., 2011).
Dentre as opções de manejo, o uso do controle químico tem sido uma alternativa viável e eficiente. Contudo, é necessária a avaliação dos herbicidas em condições de laboratório, câmaras de crescimento, casa de vegetação, microcosmos e mesocosmos (Getsinger et al., 2008).

$\mathrm{O}$ uso de herbicidas para o controle de plantas aquáticas é fundamental para o estabelecimento de estratégias de manejo. O bispyribac-sodium foi testado no controle de Sagittaria lancifolia (Glomski e Mudge, 2013); a mistura de glyphosate com saflufenacil foi utilizada no controle de Brachiaria decumbens (Queiroz et al., 2014); o 2,4-D para o Myriophyllum heterophyllum (Haug e Bellaud, 2013); o imazapyr para o controle de plantas aquáticas flutuantes (Cruz et al., 2015a); e o arylpicolinate para Alternanthera philoxeroides (Richardson et al., 2016). Outro ponto importante foi aprovação da Resolução $n^{\circ} 467$ Publicada no DOU n ${ }^{\circ} 135$, de 17 de julho de 2015 que dispõe sobre critérios para a autorização de uso de produtos ou de agentes de processos físicos, químicos ou biológicos para o controle de organismos ou contaminantes em corpos hídricos superficiais (Brasil, 2015) que passou a regulamentar o uso do controle químico em ambientes onde a necessidade de intervenção de plantas aquáticas.

Assim, devido a ocorrência desta planta invasora em diversos ambientes alagados, várzeas e rios do Brasil e as escassas as informações sobre o controle químico, novos estudos são necessários para a escolha de herbicidas e doses, além de possíveis misturas (Mudge, 2013; Mudge et al., 2014).

Para tanto, objetivo deste estudo foi avaliar a eficácia de glyphosate, saflufenacil, imazapyr, imazamox e penoxsulam, isolados e em mistura, para o controle de braquiária d'agua (U. subquadripara).

\section{Material e métodos}

As plantas foram coletadas em uma área de várzea, com $20^{\circ} 35^{\prime} 16,66^{\prime \prime}$ de latitude e 48 32'21,24" de longitude, localizada no município de Barretos, São Paulo. 
Após a coleta, as plantas foram acondicionadas em casa de vegetação, onde cinco ponteiros de $15 \mathrm{~cm}$ de comprimento foram transferidos para caixas plásticas de $15,5 \times 15,5 \mathrm{~cm}$ e $10,5 \mathrm{~cm}$ de profundidade, contendo $5 \mathrm{~cm}$ de solo, areia e substrato orgânico (na proporção 1:1:1 V V${ }^{-1}$ ), e foi fornecida lâmina de água de $2 \mathrm{~cm}$. As plantas foram cultivadas por 30 dias (folhas desenvolvidas e colmo ereto) até o momento da aplicação dos herbicidas.

Os quatros experimentos foram conduzidos em casa de vegetação com temperatura entre 25 e $32{ }^{\circ} \mathrm{C}$, fotoperíodo natural (maio a setembro) e umidade relativa entre 40 e $65 \%$. $\mathrm{O}$ delineamento experimental foi o inteiramente casualizado (DIC), com cinco repetições por tratamento e um controle (testemunha - sem aplicação dos herbicidas). As aplicações foram realizadas com pulverizador costal à pressão constante de $25 \mathrm{psi}$, mantida por $\mathrm{CO}_{2}$, equipado de barra com pontas de jato plano leque DG 11002 $\left(\right.$ Teejet $^{\circledR}$ ), espaçadas em $50 \times 50 \mathrm{~cm}$, com volume de calda equivalente a $200 \mathrm{~L} \mathrm{ha}^{-1}$.

Os herbicidas utilizados neste estudo foram o glyphosate (480 g i.a L ${ }^{-1}$, Rodeo ${ }^{\circledR}$ ), imazamox (700 g i.a. kg-1 $\mathrm{Sweeper}^{\circledR}$ ), saflufenacil (700 g i.a. kg-1 Heat $\left.^{\circledR}\right)$, imazapyr (266,3 g i.a L ${ }^{-1}$, Contain $\left.^{\circledR}\right)$ e penoxsulam (240 g i.a L ${ }^{-1}$, Ricer $\left.^{\circledR}\right)$. A relação dos experimentos, bem como doses utilizadas para cada tratamento está descrita na Tabela 1.
As avaliações foram efetuadas aos $7,15,21,30,45$ e 60 dias após a aplicação por notas de eficácia (três avaliadores) segundo a escala proposta pela SBCPD (1995) e ajustado o conceito de controle de 0 a $20 \%$ (nenhum controle - E); de 21 a 40\% (controle considerado regular - D); de 41 a $60 \%$ (controle suficiente ou bom - C); de 81 a $90 \%$ (controle satisfatório - B); de 81 a $100 \%$ (controle excelente - A).

Os dados de porcentagens de controle foram submetidos a análise de variância (ANOVA) e suas médias foram testadas pelo Teste de Tukey a 5\% pelo software AgroEst ${ }^{\circledR}$ (Barbosa e Maldonado Junior, 2015).

\section{Resultados e discussão}

No experimento 1 , em 3 DAA a maior eficácia ocorreu em Imazamox $140 \mathrm{~g} \mathrm{ha}^{-1}$ com $25 \%$ de controle, diferido significativamente dos demais tratamentos (Tabela 2). Em 7 DAA as maiores eficácias ocorrem com as misturas de imazamox $35+$ glyphosate $720 \mathrm{~g} \mathrm{ha}^{-1} \mathrm{e}$ imazamox $25+$ glyphosate $720 \mathrm{~g} \mathrm{ha}^{-1}$, com 75 e $70 \%$ de controle, respectivamente, diferindo dos demais tratamentos (Tabela 2). O uso das misturas acelerou o processo de sinais de toxicidade dos herbicidas com aumento de clorose e necrose de bordas de folhas. Em 15 DAA atingiram 95\% de controle os tratamentos com $1440 \mathrm{~g} \mathrm{ha}^{-1} \mathrm{de}$ glyphosate e todas as misturas, diferindo dos

Tabela 1. Doses dos herbicidas testadas nos experimentos de controle de U. subquadripara.

\begin{tabular}{|c|c|}
\hline Experimento 1 & Doses (g i.a. ha ${ }^{-1}$ ) \\
\hline Glyphosate & $240 ; 720 ;$ e 1440 \\
\hline Imazamox & 70 e 140 \\
\hline Imazamox + glyphosate & $35+720 ; 25+720 ;$ e $17+720$ \\
\hline Experimento 2 & Doses (g i.a. ha-1) \\
\hline Saflufenacil & $84 ; 112 ; 140 ;$ e 160 \\
\hline Saflufenacil + Glyphosate & $42+240 ;+84+240 ; 42+480 ; 84+480 ; 42+720 ;$ e $84+720$ \\
\hline Experimento 3 & Doses (g i.a. ha-1) \\
\hline Imazapyr & 66,$5 ; 133,1 ;$ e 213 \\
\hline Imazapyr + glyphosate & $31,9+240 ; 66,5+240 ; 133,1+240 ; 31,9+480 ; 66,5+480 ;$ e $133,1+480$ \\
\hline Experimento 4 & Doses (g i.a. ha $\left.{ }^{-1}\right)$ \\
\hline Penoxsulam & $6 ; 12 ; 24 ;$ e 48 \\
\hline Penoxulam + Glyphosate & $6+240 ; 12+240 ; 24+240 ; 6+480 ; 12+480 ;$ e $24+480$ \\
\hline
\end{tabular}


Tabela 2. Controle percentual de U. subquadripara com glyphosate e imazamox isolados e mistura durante o período experimental (Experimento 1).

\begin{tabular}{lccccccc}
\hline \multirow{2}{*}{ Tratamentos $\left(\mathbf{g ~ h a}^{-1}\right)$} & \multicolumn{7}{c}{ Dias após aplicação (DAA) } \\
\cline { 2 - 7 } & $\mathbf{3}$ & $\mathbf{7}$ & $\mathbf{1 5}$ & $\mathbf{2 1}$ & $\mathbf{3 0}$ & $\mathbf{4 5}$ & $\mathbf{6 0}$ \\
\hline Glyphosate 240 & $15 \mathrm{c}$ & $30 \mathrm{e}$ & $50 \mathrm{~d}$ & $75 \mathrm{c}$ & $95 \mathrm{~b}$ & $95 \mathrm{~b}$ & $95 \mathrm{~b}$ \\
Glyphosate 720 & $15 \mathrm{c}$ & $40 \mathrm{c}$ & $80 \mathrm{~b}$ & $95 \mathrm{a}$ & $95 \mathrm{~b}$ & $95 \mathrm{~b}$ & $95 \mathrm{~b}$ \\
Glyphosate 1440 & $20 \mathrm{~b}$ & $50 \mathrm{c}$ & $95 \mathrm{a}$ & $95 \mathrm{a}$ & $100 \mathrm{a}$ & $100 \mathrm{a}$ & $100 \mathrm{a}$ \\
Imazamox 70 & $5 \mathrm{e}$ & $10 \mathrm{f}$ & $40 \mathrm{e}$ & $40 \mathrm{~d}$ & $40 \mathrm{c}$ & $40 \mathrm{c}$ & $40 \mathrm{c}$ \\
Imazamox 140 & $25 \mathrm{a}$ & $40 \mathrm{c}$ & $60 \mathrm{c}$ & $80 \mathrm{~b}$ & $95 \mathrm{~b}$ & $95 \mathrm{~b}$ & $95 \mathrm{~b}$ \\
Imazamox 35 + Glyphosate 720 & $20 \mathrm{~b}$ & $75 \mathrm{a}$ & $95 \mathrm{a}$ & $95 \mathrm{a}$ & $100 \mathrm{a}$ & $100 \mathrm{a}$ & $100 \mathrm{a}$ \\
Imazamox 25 + Glyphosate 720 & $20 \mathrm{~b}$ & $70 \mathrm{a}$ & $95 \mathrm{a}$ & $95 \mathrm{a}$ & $100 \mathrm{a}$ & $100 \mathrm{a}$ & $100 \mathrm{a}$ \\
Imazamox 17 + Glyphosate 720 & $10 \mathrm{~d}$ & $60 \mathrm{~b}$ & $95 \mathrm{a}$ & $95 \mathrm{a}$ & $100 \mathrm{a}$ & $100 \mathrm{a}$ & $100 \mathrm{a}$ \\
\hline
\end{tabular}

Letras minúsculas diferentes na coluna indicam diferença entre os tratamentos pelo teste de Tukey $(\mathrm{p}<0,05)$.

demais tratamentos. Em 21 DAA este padrão de controle se manteve nos tratamentos acima citados e com glyphosate $720 \mathrm{~g} \mathrm{ha}^{-1}$ (Tabela 2). A partir de 30 DAA, com $1440 \mathrm{~g} \mathrm{ha}^{-1}$ de glyphosate ocorreu $100 \%$ de controle de $U$. subquadripara, diferindo significativamente dos demais tratamentos com aplicação do glyphosate e imazamox isolados (Tabela 2).

A melhor eficácia $\left(1440 \mathrm{~g} \mathrm{ha}^{-1}\right)$ foi similar ao ocorrido em condições semelhantes de estudo com $100 \%$ de controle desta mesma planta com a aplicação de $2880 \mathrm{~g} \mathrm{ha}^{-1}$ de glyphosate, na formulação Rodeo ${ }^{\circledR}+0,5 \%$ de adjuvante Aterbane $\mathrm{BR}^{\circledR}$ na calda (Cruz et al., 2015b).

Para o imazamox ocorreu controle de 95\% (excelente), a partir de 30 DAA na dose de $140 \mathrm{~g} \mathrm{ha}^{-1}$, com diferença significativa em relação a $70 \mathrm{~g} \mathrm{ha}^{-1}$, com nota de controle insatisfatório em todos os períodos de avaliação (Tabela 2). A eficácia na dose de $140 \mathrm{~g} \mathrm{ha}^{-1}$ foi similar ao imazamox no controle de Zostera japonica na dose de $0,56 \mathrm{~kg} \mathrm{ha}^{-1}$ (Patten, 2015), porém isolado este herbicida, na dose de $70 \mathrm{~g}$ i.a ha ${ }^{-1}$, não foi efetivo na redução de biomassa fresca e massa seca de Eichhornia crassipes e Pistia stratiotes (Mudge e Netherland, 2014).

A aplicação da mistura de herbicidas (imazamox + glyphosate) permitiu a redução de $50 \%$ da dose efetiva (1440 $\left.\mathrm{g} \mathrm{ha}^{-1}\right)$ de glyphosate para $720 \mathrm{~g} \mathrm{ha}^{-1}$ e da dose mais efetiva de imazamox de $140 \mathrm{~g} \mathrm{ha}^{-1}$ para $35 \mathrm{~g} \mathrm{ha}^{-1}$, com diferença significativa em relação aos demais tratamentos e similar ao glyphosate $\left(1440 \mathrm{~g} \mathrm{ha}^{-1}\right)$, em 15 DAA, com aumento na eficácia de $95 \%$ e 100\% em 30 DAA e similar também a eficácia do glyphosate $720 \mathrm{~g} \mathrm{ha}^{-1}$ e 25 ou $17 \mathrm{~g} \mathrm{ha}^{-1}$ de imazamox (Tabela 2), com excelente nota controle de U subquadripara. A eficácia obtida neste estudo foi similar ao imazamox, na dose de $560 \mathrm{~g} \mathrm{ha}^{-1}$ com controle visual de $100 \%$ de erva de Jacaré (Alternanthera peploides) e $81 \%$ de Myriophyllum aquaticum (Emerine et al., 2010). Outras misturas de imazamox com carfentrazone $\left(16,6\right.$ g i.a ha $\left.{ }^{-1}\right)$ e flumioxazin $(17,9$ g i.a ha-1 $)+o$ surfactante óleo vegetal $\left(1,0 \% \mathrm{v} \mathrm{v}^{-1}\right)$ também promoveram melhor desempenho na redução de biomassa fresca e seca de Eichhornia crassipes e Pistia stratiotes (Mudge e Netherland, 2014), similar ao efeito ocorrido neste estudo indicado o efeito positivo da mistura na eficácia.

No experimento 2, em todos os períodos avaliados, as misturas apresentaram eficácia superior a aplicação de saflufenacil isolado (Tabela 3). A utilização de saflufenacil, nas doses de 84 a $168 \mathrm{~g} \mathrm{ha}^{-1}$, não exibiu eficácia satisfatória, similar ao descrito em arroz (Oryza sativa), outra Poaceae, nas doses de 12,5 a $50 \mathrm{~g} \mathrm{ha}^{-1} \mathrm{com}$ apenas $26 \%$ de toxicidade (clorose e necrose) das plantas (Camargo et al., 2011) e inferior a eficácia para Sagittaria monteuindensis em 75 a $150 \mathrm{~g} \mathrm{ha}^{-1}$ de saflufenacil com três adjuvantes 
Tabela 3. Controle percentual de U. subquadripara com saflufenacil e glyphosate isolados e em mistura durante o período experimental (Experimento 2).

\begin{tabular}{lccccccc}
\hline \multirow{2}{*}{ Tratamentos $\left(\mathbf{g ~ h a}^{-1}\right)$} & \multicolumn{7}{c}{ Dias após aplicação (DAA) } \\
\cline { 2 - 8 } & $\mathbf{3}$ & $\mathbf{7}$ & $\mathbf{1 5}$ & $\mathbf{2 1}$ & $\mathbf{3 0}$ & $\mathbf{4 5}$ & $\mathbf{6 0}$ \\
\hline Saflufenacil 84 & $5 \mathrm{c}$ & $10 \mathrm{~d}$ & $15 \mathrm{c}$ & $30 \mathrm{~b}$ & $30 \mathrm{c}$ & $30 \mathrm{~b}$ & $30 \mathrm{~b}$ \\
Saflufenacil 112 & $5 \mathrm{c}$ & $10 \mathrm{~d}$ & $15 \mathrm{c}$ & $30 \mathrm{~b}$ & $30 \mathrm{c}$ & $30 \mathrm{~b}$ & $30 \mathrm{~b}$ \\
Saflufenacil 140 & $5 \mathrm{c}$ & $10 \mathrm{~d}$ & $15 \mathrm{c}$ & $30 \mathrm{~b}$ & $30 \mathrm{c}$ & $30 \mathrm{~b}$ & $30 \mathrm{~b}$ \\
Saflufenacil 168 & $5 \mathrm{c}$ & $10 \mathrm{~d}$ & $15 \mathrm{c}$ & $30 \mathrm{~b}$ & $30 \mathrm{c}$ & $30 \mathrm{~b}$ & $30 \mathrm{~b}$ \\
Saflufenacil 42 + Glyphosate 240 & $15 \mathrm{~b}$ & $30 \mathrm{c}$ & $60 \mathrm{c}$ & $80 \mathrm{a}$ & $90 \mathrm{a}$ & $90 \mathrm{a}$ & $90 \mathrm{a}$ \\
Saflufenacil 84 + Glyphosate 240 & $15 \mathrm{~b}$ & $50 \mathrm{~b}$ & $80 \mathrm{a}$ & $90 \mathrm{a}$ & $90 \mathrm{a}$ & $90 \mathrm{a}$ & $90 \mathrm{a}$ \\
Saflufenacil 42 + Glyphosate 480 & $20 \mathrm{a}$ & $55 \mathrm{ab}$ & $95 \mathrm{a}$ & $95 \mathrm{a}$ & $95 \mathrm{a}$ & $100 \mathrm{a}$ & $100 \mathrm{a}$ \\
Saflufenacil 84 + Glyphosate 240 & $20 \mathrm{a}$ & $50 \mathrm{~b}$ & $90 \mathrm{a}$ & $95 \mathrm{a}$ & $95 \mathrm{a}$ & $100 \mathrm{a}$ & $100 \mathrm{a}$ \\
Saflufenacil 42 + Glyphosate 720 & $25 \mathrm{a}$ & $65 \mathrm{ab}$ & $95 \mathrm{a}$ & $95 \mathrm{a}$ & $95 \mathrm{a}$ & $100 \mathrm{a}$ & $100 \mathrm{a}$ \\
Saflufenacil 84 + Glyphosate 720 & $25 \mathrm{a}$ & $70 \mathrm{a}$ & $95 \mathrm{a}$ & $95 \mathrm{a}$ & $95 \mathrm{a}$ & $100 \mathrm{a}$ & $100 \mathrm{a}$ \\
\hline
\end{tabular}

Letras minúsculas diferentes na coluna indicam diferença entre os tratamentos pelo teste de Tukey $(\mathrm{p}<0,05)$.

$\left(\right.$ Dash $^{\circledR} \mathrm{HC}$, Assist $^{\circledR}$ e Veget Oil $\left.{ }^{\circledR}\right)$, com controle máximo de $70 \%$ com $150 \mathrm{~g} \mathrm{ha}^{-1}+$ Veget Oil $^{\circledR} 1 \mathrm{~L} \mathrm{ha}^{-1}$ (Moura et al., 2016).

$\mathrm{Na}$ aplicação de saflufenacil + glyphosate, a utilização de $240 \mathrm{~g} \mathrm{ha}^{-1}$ de glyphosate e $42 \mathrm{~g} \mathrm{ha}^{-1}$ de saflufenacil ocorreu eficácia de $90 \%$ a partir de 30 DAA e 240 ha $^{-1}+84 \mathrm{~g} \mathrm{ha}^{-1}$ o mesmo controle a partir de 21 DAA, diferindo significativamente da aplicação do herbicida isolado (Tabela 3). Para os períodos de avaliação não ocorreu diferença significativa para as misturas de herbicidas a partir de 30 DAA (Tabela 2).

No experimento 1 (Tabela 2) a dose de $240 \mathrm{~g} \mathrm{ha}^{-1}$ glyphosate isolado apresentou o máximo de $95 \%$ de controle entre 21 e 60 DAA com as plantas com os caules com coloração verdes e algumas folhas ainda presentes similar ao 42 e $84 \mathrm{~g} \mathrm{ha}^{-1}$ de saflufenacil + glyphosate (240 e $\left.480 \mathrm{~g} \mathrm{ha}^{-1}\right)$, com o máximo de $90 \%$ de controle aos 60 DAA (Tabela 3). Estas doses de saflufenacil acrescidas de $720 \mathrm{~g} \mathrm{ha}^{-1}$ promoveram $100 \%$ de controle da $U$. subquadripara (Tabela 3), sendo mais efetivas do que o glyphosate na formulação Rodeo ${ }^{\circledR}$ com controle desta mesma planta com 2880 g i.a ha ${ }^{-1}$ (Cruz et al., 2015b) e do glyphosate 3360 e $4320 \mathrm{~g} \mathrm{ha}^{-1}$ para a U. subquadripara e U. mutica (Carbonari et al., 2003), enquanto que, para Brachiaria decumbens a mistura de $1080 \mathrm{~g}$ i.a de glyphosate $+24,5 \mathrm{~g} \mathrm{ha}^{-1}$ de saflufenacil apresentou 99,3\% de controle em 14 DAA (Queiroz et al., 2014).

No experimento 3, todas as doses de imazapyr apresentou controle satisfatório entre 80 e $85 \%$ em apenas 60 DAA, eficácia inferior e com diferença significativa em relação as misturas (Tabela 4). A eficácia deste herbicida isolado difere do mesmo para E crassipes e P stratiotes com 95 a 100\% de controle, com a utilização de 932 e 1065,2 $\mathrm{g} \mathrm{ha}^{-1}$ (Cruz et al., 2015b) e glyphosate com eficácia de $100 \%$ no controle e $U$. subquadripara com a dose 1597,8 $\mathrm{g} \mathrm{ha}^{-1}$ em 60 DAA (Cruz et al., 2015a).

A mistura de 31,9 $\mathrm{g} \mathrm{ha}^{-1}$ de imazapyr $+240 \mathrm{~g} \mathrm{ha}^{-1}$ de glyphosate $\left(50 \%\right.$ da dose de $66,5 \mathrm{~g} \mathrm{ha}^{-1}$ com eficácia de $80 \%$ ) aumentou significativamente para 90\% (nota de eficácia ainda considera satisfatória), em relação ao imazapyr isolado em 60 DAA(Tabela 4). Em imazapyr 31,9, 66,5 e $213 \mathrm{~g} \mathrm{ha}^{-1}+240$ e $480 \mathrm{~L} \mathrm{ha}^{-1}$ de glyphosate ocorreu $100 \%$ de controle da U. subquadripara, sem diferença significativa entre as misturas, a partir de 45 DAA (Tabela 4), diferindo da eficácia obtida para Arundo donax com 270,7 g ha $^{-1}$ de imazapyr (formulação Habitat ${ }^{\circledR}$ ) em aplicação mensal aérea via helicóptero durante um ano, este não apresentou controle visual com uma pequena diminuição de atividade de clorofila na primavera (Spencer et al., 2009), entretanto, a utilização da mistura de imazapyr + glyphosate possibilitou o aumento da efetividade de controle 
Tabela 4. Controle percentual de U. subquadripara com imazapyr e glyphosate isolados e em mistura durante o período experimental (Experimento 3).

\begin{tabular}{lccccccc}
\hline \multirow{2}{*}{ Tratamentos $\left(\mathbf{g ~ h a}^{-1}\right)$} & \multicolumn{7}{c}{ Dias após aplicação (DAA) } \\
\cline { 2 - 8 } & $\mathbf{3}$ & $\mathbf{7}$ & $\mathbf{1 5}$ & $\mathbf{2 1}$ & $\mathbf{3 0}$ & $\mathbf{4 5}$ & $\mathbf{6 0}$ \\
\hline Imazapyr 66,5 & $0 \mathrm{~d}$ & $5 \mathrm{e}$ & $10 \mathrm{e}$ & $50 \mathrm{~d}$ & $50 \mathrm{f}$ & $65 \mathrm{~d}$ & $80 \mathrm{c}$ \\
Imazapyr 133,1 & $0 \mathrm{~d}$ & $5 \mathrm{e}$ & $10 \mathrm{e}$ & $50 \mathrm{~d}$ & $60 \mathrm{e}$ & $70 \mathrm{c}$ & $85 \mathrm{c}$ \\
Imazapyr 213 & $5 \mathrm{c}$ & $10 \mathrm{~d}$ & $30 \mathrm{~d}$ & $50 \mathrm{~d}$ & $60 \mathrm{e}$ & $70 \mathrm{c}$ & $85 \mathrm{c}$ \\
Imazapyr 31,9+ Glyphosate 240 & $20 \mathrm{~b}$ & $30 \mathrm{c}$ & $30 \mathrm{~d}$ & $45 \mathrm{e}$ & $70 \mathrm{~d}$ & $80 \mathrm{~b}$ & $90 \mathrm{~b}$ \\
Imazapyr 66,5 + Glyphosate 240 & $20 \mathrm{~b}$ & $30 \mathrm{c}$ & $50 \mathrm{c}$ & $55 \mathrm{c}$ & $75 \mathrm{c}$ & $95 \mathrm{a}$ & $100 \mathrm{a}$ \\
Imazapyr 133,1 + Glyphosate 240 & $25^{\mathrm{a}}$ & $35 \mathrm{~b}$ & $70 \mathrm{~b}$ & $80 \mathrm{~b}$ & $90 \mathrm{~b}$ & $95 \mathrm{a}$ & $100 \mathrm{a}$ \\
Imazapyr 31,9+ Glyphosate 480 & $25^{\mathrm{a}}$ & $55 \mathrm{a}$ & $80 \mathrm{a}$ & $95 \mathrm{a}$ & $95 \mathrm{a}$ & $95 \mathrm{a}$ & $100 \mathrm{a}$ \\
Imazapyr 66,5 + Glyphosate 480 & $25 \mathrm{a}$ & $55 \mathrm{a}$ & $80 \mathrm{a}$ & $95 \mathrm{a}$ & $95 \mathrm{a}$ & $95 \mathrm{a}$ & $100 \mathrm{a}$ \\
Imazapyr 133,1 + Glyphosate 480 & $25 \mathrm{a}$ & $55 \mathrm{a}$ & $90 \mathrm{a}$ & $95 \mathrm{a}$ & $95 \mathrm{a}$ & $95 \mathrm{a}$ & $100 \mathrm{a}$ \\
\hline
\end{tabular}

Letras minúsculas diferentes na coluna indicam diferença entre os tratamentos pelo teste de Tukey $(\mathrm{p}<0,05)$.

Tabela 5. Controle percentual de U. subquadripara com penoxsulam e glyphosate isolados e em mistura durante o período experimental (Experimento 4).

\begin{tabular}{|c|c|c|c|c|c|c|c|}
\hline \multirow{2}{*}{ Tratamentos $\left(\mathrm{g} \mathrm{ha}^{-1}\right)$} & \multicolumn{7}{|c|}{ Dias após aplicação (DAA) } \\
\hline & 3 & 7 & 15 & 21 & 30 & 45 & 60 \\
\hline Penoxsulam 6 & $5 b$ & $5 c$ & $25 b$ & $25 c$ & $25 c$ & $25 c$ & $25 c$ \\
\hline Penoxsulam 12 & $5 b$ & $5 c$ & $25 b$ & $25 c$ & $25 c$ & $25 c$ & $25 \mathrm{c}$ \\
\hline Penoxsulam 24 & $5 b$ & $10 \mathrm{~b}$ & $25 b$ & $25 \mathrm{c}$ & $25 \mathrm{c}$ & $25 \mathrm{c}$ & $25 \mathrm{c}$ \\
\hline Penoxsulam 36 & $5 b$ & $10 \mathrm{~b}$ & $25 b$ & $25 \mathrm{c}$ & $25 c$ & $25 c$ & $25 \mathrm{c}$ \\
\hline Penoxsulam 48 & $5 b$ & $10 \mathrm{~b}$ & $25 b$ & $45 b$ & $60 \mathrm{~b}$ & $70 \mathrm{~b}$ & $80 \mathrm{c}$ \\
\hline Penoxsulam 6 + Glyphosate 240 & $30 \mathrm{a}$ & $50 \mathrm{a}$ & $95 \mathrm{a}$ & $95 \mathrm{a}$ & $95 \mathrm{a}$ & $95 \mathrm{a}$ & $100 \mathrm{a}$ \\
\hline Penoxsulam $12+$ Glyphosate 240 & $30 \mathrm{a}$ & $50 \mathrm{a}$ & $95 \mathrm{a}$ & $95 \mathrm{a}$ & $95 \mathrm{a}$ & $95 \mathrm{a}$ & $100 \mathrm{a}$ \\
\hline Penoxsulam 24 + Glyphosate 240 & $30 \mathrm{a}$ & $50 \mathrm{a}$ & $95 \mathrm{a}$ & $95 \mathrm{a}$ & $95 \mathrm{a}$ & $95 \mathrm{a}$ & $100 \mathrm{a}$ \\
\hline Penoxsulam 6 + Glyphosate 480 & $30 \mathrm{a}$ & $50 \mathrm{a}$ & $95 \mathrm{a}$ & $95 \mathrm{a}$ & $95 \mathrm{a}$ & $95 \mathrm{a}$ & $100 \mathrm{a}$ \\
\hline Penoxsulam $12+$ Glyphosate 480 & $30 \mathrm{a}$ & $50 \mathrm{a}$ & $95 \mathrm{a}$ & $95 \mathrm{a}$ & $95 \mathrm{a}$ & $95 \mathrm{a}$ & $100 \mathrm{a}$ \\
\hline Penoxsulam 24 + Glyphosate 480 & $30 \mathrm{a}$ & $50 \mathrm{a}$ & $95 \mathrm{a}$ & $95 \mathrm{a}$ & $95 \mathrm{a}$ & $95 \mathrm{a}$ & $100 \mathrm{a}$ \\
\hline
\end{tabular}

Letras minúsculas diferentes na coluna indicam diferença entre os tratamentos pelo teste de Tukey $(p<0,05)$.

com diminuição de $2640 \mathrm{~g} \mathrm{ha}^{-1}$ de glyphosate e de $932 \mathrm{~g} \mathrm{ha}^{-1}$ de imazapyr.

A vantagem da utilização da mistura de imazapyr + glyphosate no controle de U. subquadripara pode ser atribuída a lenta morte da planta e gradativa liberação de nutrientes para o corpo hídrico, sendo a degradação e rápida liberação de nutrientes para o ambiente uma das principais críticas da utilização do controle químico de plantas daninhas aquáticas, além da possibilidade de efeito a qualidade de água, fitoplâncton e zooplâncton e outras comunidades de plantas aquáticas (Sethi et al., 2017).
O penoxsulam, na dose de $48 \mathrm{~g} \mathrm{ha}^{-1}$, apresentou eficácia de 80\%, em 60 DAA com diferença significativa em relação as demais doses aplicadas isoladamente. As demais doses isoladas não diferiram entre si (Tabela 5). A eficácia de controle para $U$. subquadripara foi inferior a eficácia deste mesmo herbicida com 94 a 100\% de controle das plantas invasoras (Echinochloa oryzoides e E. phyllopogon) com 20 a 40 g i.a ha ${ }^{-1}$ (Damalas et al., 2006) e da redução da produção de raiz e caule de Pontederia cordata e Sagittaria lanciofolia nas doses de 25, 150 e $300 \mu \mathrm{g} \mathrm{L}^{-1}$, entretanto neste estudo também foi verificado que 
as monocotiledôneas Paspalidium geminatum e Panicum hemitimon foram tolerantes ao penoxsulam (Koschnick et al., 2007), similar a todas as doses testadas 6 a $48 \mathrm{~g} \mathrm{ha}^{-1}$ neste estudo); e eficácia de $93 \%$ (temperatura $21^{\circ} \mathrm{C}$ ) no controle de Alternanthera philoxeroides (Willingham et al., 2008).

A mistura de penoxsulam + glyphosate promoveu aumento significativo no controle de $U$. subquadripara a partir de 15 DAA, com eficácia de 95\% (Tabela 5). A partir desta avaliação não ocorreu diferença significativa entre os tratamentos em todos os períodos avaliados até 45 DAA. Em 60 DAA todas as misturas testadas apresentaram $100 \%$ de controle e diferiram do penoxsulam aplicado isoladamente (Tabela 4).

Os herbicidas glyphosate e imazamox, aplicados de forma isolada, apresentaram excelente controle $(100 \%)$ da planta invasora U. subquadripara, enquanto que, o imazapyr (todas as doses testadas) e o penoxsulam ( $\left.48 \mathrm{~g} \mathrm{ha}^{-1}\right)$ apresentaram controle satisfatório com 80 a $85 \%$ de eficácia de controle. O saflufenacil, nas doses testadas, apresentou controle regular (30\%). As misturas de imazamox, saflufenacil, imazapyr e penoxsulam com o glyphosate apresentaram excelente controle em todas as condições testadas (de 81 a 100\%).

No caso específico da mistura de imazapyr e penoxsulam + glyphosate o efeito herbicida lento pode ser um fator positivo na tomada de decisão para o uso em ambientes aquáticos, várzeas, canais de transporte de água, pois pode favorecer a lenta liberação de nutrientes no ambiente, sem alterar sua dinâmica de funcionamento e auxiliar na manutenção da condição natural com aumento da diversidade de composição de plantas aquáticas (Coutris et al., 2011).

\section{Conclusões}

Os herbicidas glyphosate e imazamox isolados, apresentaram excelente controle de U. subquadripara, enquanto que, o imazapyr e o penoxsulam $\left(48 \mathrm{~g} \mathrm{ha}^{-1}\right)$ apresentaram controle satisfatório. Os herbicidas glyphosate, imazamox e imazapyr (isolados e em mistura), o saflufenacil + glyphosate (mistura) e o penoxsulam + glyphosate (mistura) podem ser empregados em programas de manejo desta planta aquática exótica e invasora. As misturas de imazamox e saflufenacil com glyphosate poderiam ser utilizadas em locais onde a liberação de nutrientes pela decomposição da planta não fosse limitante ou em locais de reinfestação após o primeiro manejo (manejo sequencial).

\section{Agradecimentos}

Os autores agradecem o programa PIBIC/CNPq (2015-2016) (Conselho Nacional de Desenvolvimento Científico e Tecnológico) pela bolsa de iniciação científica do primeiro autor.

\section{Referências}

Anda, A.; Soos, G.; Silva, J.T.; Kozma-Bogna, V. Regional evapotranspiration from a wtland incentral euroupe, In a LG-year period without human intervention. Agricultural and Forest Meteorology, v.205, n.1, p.60-72, 2015. http:// dx.doi.org/10.1016/j.agrformet.2015.02.010.

Barbosa, J.C.; Maldonado Junior, W. Agroestat ${ }^{\circledR}$ : sistema para analises estatísticas e ensaios agronômicos. Jaboticabal: UNESP, 2015.

Brasil. CONAMA - Conselho Nacional do Meio Ambiente. Resolução no 467, de 16 de julho de 2015. Diário Oficial [da] República Federativa do Brasil, Brasília, DF, 17 jul. 2015. Seção 1, p.70-71.

Bunn, S.E.; Davies, P.M.; Kellaway, D.M.; Prosser, I.P. Influence of invasive macrophytes on channel morphology and hydrology in an open tropical lowland stream, and potential control by riparian shading. Freshwater Biology, v.39, n.1, p.171-178, 1998. http://dx.doi.org/10.1046/j.13652427.1998.00264.x.

Camargo, E.R.; Senseman, S.A.; Mccauley, G.N.; Guice, J.B. Rice tolerance to saflufenacil in clomazone wees control progam. International 
Journal of Agronomy, v.2011, p.1-8, 2011. http:// dx.doi.org/10.1155/2011/402461.

Carbonari, C.A.; Martins, D.; Terra, M.A. Controle de Brachiaria subquadripara e Brachiaria mutica através de diferentes herbicidas aplicados em pósemergência. Planta Daninha, v.21, p.79-84, 2003.

Coutris, C.; Merlina, G.; Silvestre, J.; Pinelli, E.; Elger, A. Can we predict community-wide effects of herbicides from toxicity tests on macrophyte species? Aquatic Toxicology, v.101, n.1, p.49-56, 2011. http://dx.doi.org/10.1016/j. aquatox.2010.08.017.

Cruz, C.; Silva, A.F.; Shiogiri, N.S.; Garlich, N.; Pitelli, R.A. Imazapyr herbicide efficacy on floating macrophyte control and ecotoxicology for non-target organisms. Planta Daninha, v.33, n.1, p.103-108, 2015a. http://dx.doi.org/10.1590/ S0100-83582015000100012.

Cruz, C.; Silva, A.F.; Luna, L.V.; Yamauchi, A.K.F.; Garlich, N.; Pitelli, R.A. Glyphosate effectiveness in the control of macrophytes under a greenhouse condition. Planta Daninha, v.33, n.2, p.241-247, 2015b. http://dx.doi.org/10.1590/010083582015000200009.

Damalas, C.A.; Dhima, K.V.; Eleftherohorinos, I.G. Control of early watergrass (Echinochloa Oryzoides) and late watergrass (Echinochloa Phyllopogon) with cyhalofop, clefoxydim, and penoxsulam applied alone and in mixture with broadleaf herbicides. Weed Technology, v.20, n.4, p.992-998, 2006. http://dx.doi.org/10.1614/ WT-05-140.1.

Domingos, V.D.; Martins, D.; Costa, N.V.; Marchi, S.R. Fatores ambientais na distribuição de populações de Brachiaria arrecta presentes no reservatório de Barra Bonita-SP. Planta Daninha, v.29, p.37-49, 2011.

Emerine, S.E.; Richardson, R.J.; True, S.L.; West, A.M.; Roten, L.R. Greenhouse response of six aquatic invasive weeds to imazamox. Journal of
Aquatic Plant Management, v.48, p.105-111, 2010.

Getsinger, K.D.; Netherland, M.D.; Grue, C.E.; Koschnick, T.J. Improvement intheuse of aquatic herbicides and establishment os future rescarch directions. Journal of Aquatic Plant Management, v.46, p.32-42, 2008.

Glomski, L.M.; Mudge, C.R. Effect of subsurface and foliar applications of bispyribac-sodium on water hyacinth, water lettuce, and giant salvinia. Journal of Aquatic Plant Management, v.51, p.62-65, 2013.

Haug, E.J.; Bellaud, M.D. Efficacy of 2,4-D ester on variable-leaf milfoil control for partial lake treatments in New Hampshire waterbodies. Journal of Aquatic Plant Management, v.51, p.49-52, 2013.

Koschnick, T.J.; Netherland, M.D.; Haller, W.T. Effects of three ALS-inhibitors on five emergent native plant species in Florida. Journal of Aquatic Plant Management, v.45, p.47-51, 2007.

Li, W. Environmental opportunities and constraints in the reproduction and dispersal of aquatic plants. Aquatic Botany, v.118, p.62-70, 2014. http:// dx.doi.org/10.1016/j.aquabot.2014.07.008.

Lu, J.; Bunn, S.E.; Burford, M.A. Nutrient release and uptake by littoral macrophytes during waterlevel fluctuations. The Science of the Total Environment, v.622-623, p.29-40, 2018. http:// dx.doi.org/10.1016/j.scitotenv.2017.11.199.

Moura, D.D.S.; Noldin, J.A.; Galon, L.; Schreiber, F.; Martini, A.T. Management of Sagittaria montevidensis resistant to ALS and PSII mechanism of action with saflufenacil associated with different adjuvants. Revista Brasileira de Herbicidas, v.15, n.2, p.148-156, 2016. http://dx.doi.org/10.7824/ rbh.v15i2.451.

Mudge, C.R. Impact of aquatic herbicide combinations on nontarget submersed plants. Journal of Aquatic Plant Management, v.51, p.39-44, 2013. 
Mudge, C.R.; Harms, N.E.; Nachtrieb, J.G. Interactions of herbicides, surfactants, and the giant salvinia weevil (Cyrtobagous salviniae) for control of giant salvinia (Salvinia molesta). Journal of Aquatic Plant Management, v.52, p.15-21, 2014.

Mudge, C.R.; Netherland, M.D. Response of invasive floating plants and nontarget emergent plants to foliar applications of imazamox and penoxsulam. Journal of Aquatic Plant Management, v.52, p. 1-7, 2014.

Patten, K.D. Imazamox control of invasive Japanese eelgrass (Zostera japonica): Efficacy and nontarget impacts. Journal of Aquatic Plant Management, v.53, p.185-190, 2015.

Pitelli, R.L.C.M.; Toffaneli, C.M.; Vieira, E.A.; Pitelli, R.A.; Velini, E.D. Dinâmica da comunidade de macrófitas aquáticas no reservatório de Santana, RJ. Planta Daninha, v.26, n.3, p.473480, 2008. http://dx.doi.org/10.1590/S010083582008000300001 .

Queiroz, J.R.G.; Silva Junior, A.C.; Rodrigues, A.C.P.; Martins, D. Eficiência da aplicação da mistura de glyphosate com saflufenacil sobre plantas de Brachiaria decumbens. Revista Brasileira de Herbicidas, v.13, n.1, p.1-7, 2014. http://dx.doi. org/10.7824/rbh.v13i1.255.

Richardson, R.J.; Haug, E.J.; Netherland, M.D. Response of seven aquatic plants to a new arylpicolinate herbicide. Journal of Aquatic Plant Management, v.54, p.26-31, 2016.
Riis, T. Dispersal and colonisation of plants in lowland streams: success rates and bottlenecks. Hydrobiologia, v.596, n.1, p.341-351, 2008. http://dx.doi.org/10.1007/s10750-007-9107-0.

SBCPD - Sociedade Brasileira da Ciências das Plantas Daninhas. Procedimentos para instalação, avaliação e análise de experimentos com herbicidas. Londrina, 1995. p.42.

Sethi, S.A.; Carey, M.P.; Morton, J.M.; GuerronOrejuela, E.; Decino, R.; Willette, M.; et al. Rapid response for invasive waterweeds at the arctic invasion front: Assessment of collateral impacts from herbicide treatments. Biological Conservation, v.212, p.300-309, 2017. http:// dx.doi.org/10.1016/j.biocon.2017.06.015.

Spencer, D.F.; Tan, W.; Liow, P.S.; Ksander, G.G.; Whitehand, L.C. Evaluation of a late summer imazapyr treatment for managing giant reed (Arundo donax). Journal of Aquatic Plant Management, v.47, p.40-43, 2009.

Strayer, D.L. Alien species in fresh waters: ecological effects, interactions with other stressors, and prospects for the future. Freshwater Biology, v.55, p.152-174, 2010. http://dx.doi.org/10.1111/ j.1365-2427.2009.02380.x.

Willingham, S.D.; Senseman, S.A.; McCauley, G.N.; Chandler, J.M. Effect of temperature and propanil on penoxsulam efficacy, absorption, and translacation in alligatorweed (Alternanthera philoxeroides). Weed Science, v.56, n.6, p.780784, 2008. http://dx.doi.org/10.1614/WS-08-056.1. 